\title{
Lumbar Disca Cyst as a Rare Cause of Low Badk Pain and Radiculopathy
}

\author{
Eui-Jin Cho', Bum-Joon Kim ${ }^{1 凶}$, Se-Hoon Kim ${ }^{1}$, Seung-Hwan Lee ${ }^{1}$ Hwa-Eun $\mathrm{Oh}^{2}$ \\ Departments of ${ }^{1}$ Neurosurgery, ${ }^{2}$ Pathology, Korea University Ansan Hospital, Korea University College of Medicine, Ansan, Korea
}

Discal cysts that lead to low back pain and radiculopathy are uncommon. To date, only a few cases have been reported in the literature. Herein, we report the case of a 30-year-old man who experienced low back pain and radiculopathy due to a lumbar discal cyst. On magnetic resonance images, an extradural cystic mass was seen as a low signal intensity on T1-weighted images and a high signal intensity on T2-weighted images, respectively. The cyst was a grossly oval-shaped mass with hematoma connected to the adjacent intervertebral disc. Radiculopathy and low back pain were dramatically relieved after surgical removal. As in our experience and other case reports, surgical resection can be considered as the first treatment option in patients with symptomatic discal cysts.

Key Words: Intervertebral Disc; Cysts; Radiculopathy; Low Back Pain

$\triangle$ Corresponding Author: Bum-Joon Kim, Department of Neurosurgery, Korea University Ansan Hospital, Korea University College of Medicine, 123 Jeokgeum-ro, Danwon-gu, Ansan-si, Gyeonggi-do 15355, Korea. Tel: +82-31-412-5053, Fax: +82-31-412-5054, E-mail: nsbjkim@gmail.com

\section{INTRODUCTION}

Many individuals suffer from radiculopathy, which is often caused by degenerative disc diseases, such as disc herniation or spinal stenosis. Because symptoms by degenerated disc diseases are possible to be relieved spontaneously within a few weeks, patients can be treated with nonsurgical procedures, such as nerve block or physical therapies in their early stages.

However, in some cases, radiculopathy was reported to be caused by discal cysts rather than herniations. When a patient with radiculopathy shows cystic lesion on magnetic resonance images (MRIs), the differential diagnosis should include other intradural and extradural masses, such as synovial cyst, ganglion cyst, pseudocyst, hematoma, metastatic tumor, and arachnoid cyst because several kinds of cysts can develop in the spinal $\mathrm{canal}^{10)}$. Among the intraspinal cysts, owing to its rare prevalence, the pathophysiology and clinical course of discal cysts are still obscure.

Herein, we report the case of a patient who had a lumbar discal cyst with lumbar and radiating leg pain, which were refractory to epidural steroid injection and physical therapy, treated successfully with a cyst removal surgery.

\section{CASE REPORT}

A 30-year-old male patient with lumbago and radiating pain from the left buttock to the lateral calf along the L5 dermatome visited our clinic. He had no current or past history of trauma. Although he had received several epidural steroid injections and physical therapies at another hospital for 2 months, the pain worsened and thereby interfered with his usual activities of living. The visual analog scale pain scores were 6 at back pain and 8 at radiating pain. There were also numbness and tingling sensations on the left posterior thigh and leg. Due to lumbar pain, he showed lumbar motion limitations and paravertebral tenderness on the lower lumbar level during the neurologic examination. Left ankle dorsiflexion weakness grade $4 / 5$ and L5 dermatome hypesthesia were also found. He had a positive finding at $45^{\circ}$ in the left straight leg raising test. Other motor examinations revealed normal findings.

The initial plain X-ray on the lumbar spine showed a mild intervertebral space narrowing at the L4/5 and L5/S1 levels, which was unremarkable. On magnetic resonance imaging (MRI), a $9 \mathrm{~mm} \times 5 \mathrm{~mm} \times 9 \mathrm{~mm}$-sized epidural cystic mass was found at the left subarticular area of L4/5 compressing the thecal sac and the left L5 nerve root (Fig. 1). The cyst showed an iso- to low-signal intensity on the T1-weighted image (WI) and a high-signal intensity on the T2-WI with a septated appearance (Fig. 1).

Because the patient did not show any relief of pain despite sufficient conservative therapy over 2 months, he underwent surgery. A laminotomy was performed on the left lower portion of the L4 lamina. After ligamentum flavum removal, a dark reddish cystic mass was identified at the axillary portion of 

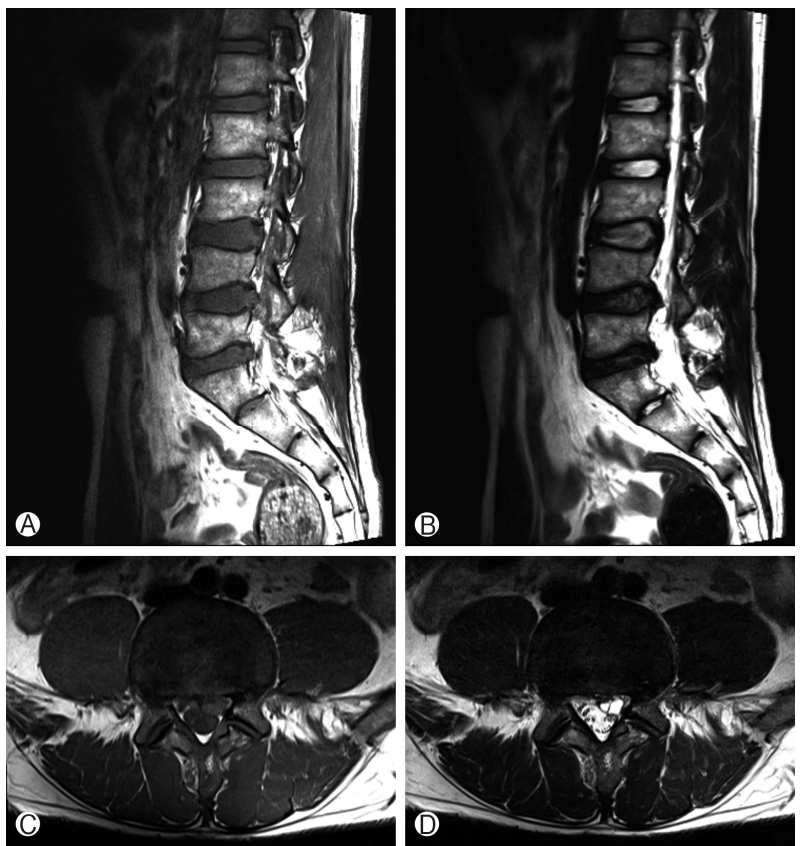

Fig. 1. Magnetic resonance images. A $9 \mathrm{~mm} \times 5 \mathrm{~mm} \times 9 \mathrm{~mm}$-sized extradural cystic mass adheres to the degenerated $L 4 / 5$ disc (A, B), which compresses the left $L 5$ nerve root at the left subarticular area (C, D). The cyst shows an iso- to low-signal intensity and high-signal intensity on the T1- (C) and T2-weighted images (D), respectively.

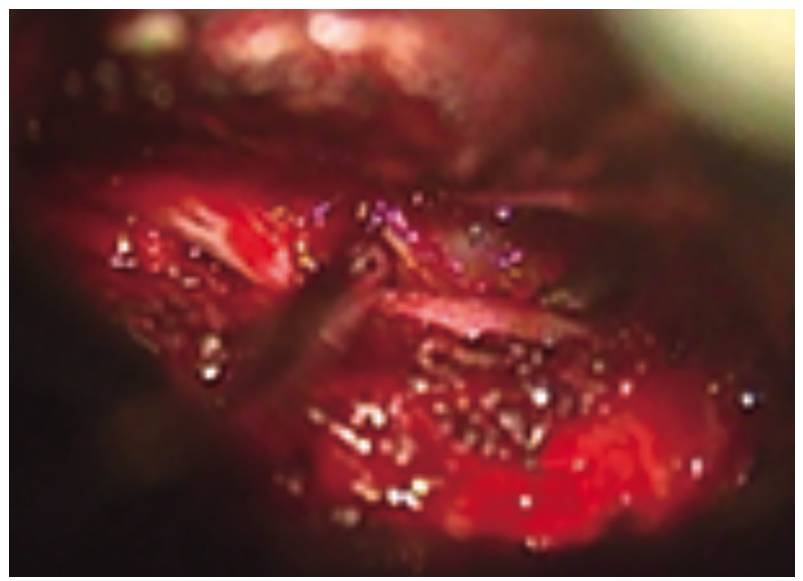

Fig. 2. Intraoperative image. A round thick-walled dark reddish cystic mass is identified at the ventral portion of the left $L 5$ nerve root. The cyst firmly adheres to the annulus fibrosus.

the left L5 nerve root (Fig. 2). The cyst showed an adhesion with the surrounding dural sac and the nerve root. When the cyst was punctured, an old blackish hematoma gushed out. The cyst was connected to the annulus fibrosus of the L4/5 disc, and the margin was obscure. After meticulous dissection, the cyst was totally resected, and the left L5 nerve root was freed. Pain on the lumbar and posterior thigh and leg decreased promptly after surgery. There was residual numbness, which

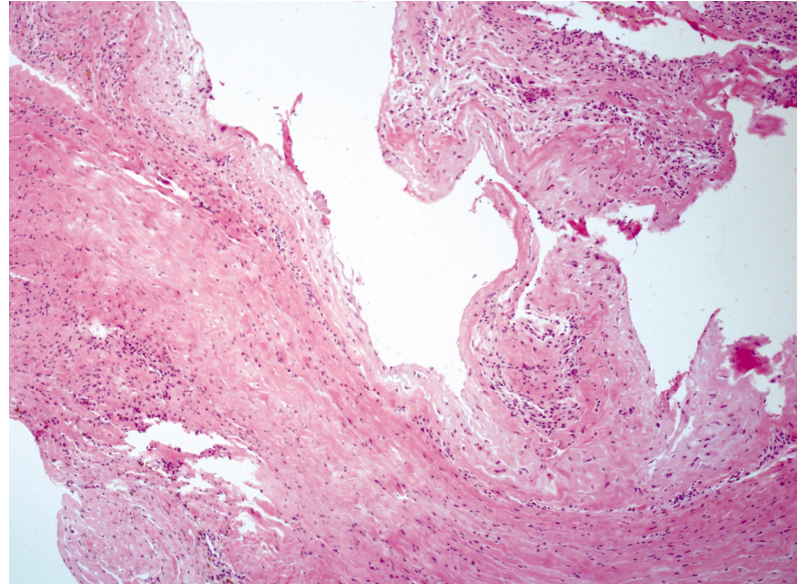

Fig. 3. Histopathologic finding. The cyst contains an old hematoma and the cyst wall consists of fibrous connective tissues without any cell lining and disc material (Hematoxylin \& Eosin stain, $\times 100)$.

was then resolved upon assessment during the follow-up 1 month later. He was diagnosed with a lumbar discal cyst based on the histopathologic features of the cyst wall consisted of fibrous connective tissues without any cell lining and disc material (Fig. 3).

\section{DISCUSSION}

A discal cyst is a rare entity causing lumbar pain and radiculopathy ${ }^{2)}$. Several cases of discal cyst have been reported to date in the literature, and the human discal cysts in all reported cases were located on the lumbar level ${ }^{1-5,9,12,14)}$, while cervical discal cysts have been reported in $\operatorname{dog} s^{7)}$. Although there is no conclusive explaination of why discal cysts in human were reported only in the lumbar area, this finding implies that the upright posture of human and axial loading may have a role in the mechanism of discal cyst formation.

The clinical manifestation of pain caused by discal cysts is similar to that caused by herniated discs that compress the thecal sac and roots ${ }^{4}$. However, the mean age at diagnosis of discal cysts in the literature was early 30 s, which was slightly younger than that of herniated disc cases; further, discal cysts were more common in men and Asians ${ }^{1,5}$. In our case, the patient was a 30-year-old man from Central Asia, and the discal cyst was located at L4-5.

MRI is the most important tool in diagnosing discal cysts. The ventrolateral extradural cystic mass is the key finding that allows differentiation from disc herniation and other intraspinal cystic lesions ${ }^{1)}$. Discal cysts usually appear as oval- to roundshaped low T1 and high T2 signal lesions on MRIs ${ }^{3,9)}$. The signal intensity is similar with that of cerebrospinal fluid, which implies that the content of the cyst is serous. The cyst wall usually shows a rim enhancement with contrast ${ }^{1)}$. In many 
cases, discal cysts are combined with herniated discs, and a discogram or MRI can often visualize communication with the involved herniated $\operatorname{disc}^{3,11)}$. In literature reviews, discal cysts occur at the lumbar level, particularly in L4-5 and L5$\mathrm{S} 1^{1-5,9,11,14)}$. Considering the similar prevalence and coexistence, there seems to be some relationship between the development of discal cysts and degeneration of the lumbar disc.

For the histopathologic finding, Chiba et al. reported a fibrous connective tissue lining of the discal cyst without specific cell layers or disc materials ${ }^{3)}$. In the current case, histopathology showed a benign connective tissue cyst wall containing an old hematoma (Fig. 3). Most features were consistent with those reported by Chiba et al..

The pathogenesis of discal cysts remains unknown to date. Several hypotheses for the genesis of discal cysts have been considered. Several authors suggest that hemorrhage occurs at the epidural venous plexus and the hematoma absorption resulted in cystic change ${ }^{3,17}$. Kono et al. hypothesized that discal cyst formation results from the degeneration of herniated discs with fluid production as in synovial cysts at the facet joint and meniscal cysts on the knees ${ }^{11)}$. Marshman et al. explained that discal cysts develop in a similar process as in ganglion cysts at the posterior longitudinal ligament or annulus fibrosis ${ }^{13)}$. However, no single hypothesis explains how discal cysts are perfectly formed considering the presence of hematoma in the cyst and connection between the stalk and cyst. In the current case, old hematoma contents was found inside the cyst though the patient had no trauma history. Considering the intraoperative finding of capsule communicating with the annulus, we suggest that hemorrhage results from the neovascularization of the degenerated annulus fibrosus rather than hemorrhage from the epidural venous plexus.

There exist several studies that imply a role of inflammatory reactions in the pathophysiology of painful radiculopathy caused by degenerative disc diseases. Elevated inflammatory enzyme levels, including phospholipase A levels, were observed at the epidural interface of the injured site, which irritate the nerve root and allow conduction block ${ }^{12,15)}$. Further, Takahashi et al. suggested that interleukin-1 was detected at the site of disc herniation ${ }^{16)}$. Previous research studies also found that noxious stimuli can yield peripheral and central sensitizations, which convert pain from acute to chronic ${ }^{6}$. Therefore, if the lumbago or radiating pain of a patient does not resolve despite proper conservative therapy, performing surgical interventions could be beneficial by elimination of inflammatory cytokines and noxious stimuli.

In most reported cases, the pain and symptoms of patients after surgical removal of discal cysts were relieved ${ }^{1,2,5,9,12,14)}$. Matsumoto et al. reported their successful result using a minimally invasive microendoscopic resection of a discal cyst ${ }^{14}$. In the present case, the discal cyst was easily resected using a microscopic approach, although there was an adhesion.
Although there exists a report that a discal cyst was treated by a computed tomography-guided aspiration and steroid injection $^{10)}$, concerning the regrowth of the cyst due to microbleeding from the vascularized capsule, we recommend total resection of the cyst.

\section{CONCLUSION}

A discal cyst is an extremely rare cause of lumbar pain and radiculopathy. Patients with discal cysts show similar clinical manifestations as in herniated discs. Once discal cysts are diagnosed via a meticulous review of MRIs, a gross total resection of the cyst can provide dramatic relief of the pain.

\section{REFERENCES}

1. Aydin S, Abuzayed B, Yildirim H, Bozkus H, Vural M: Discal cysts of the lumbar spine: report of five cases and review of the literature. Eur Spine J 19:1621-1626, 2010

2. Certo F, Visocchi M, Borderi A, Pennisi C, Albanese V, Barbagallo GM: Lumbar intervertebral discal cyst: a rare cause of low back pain and radiculopathy. Case report and review of the current evidences on diagnosis and management. Evid Based Spine Care J 5:141-148, 2014

3. Chiba K, Toyama Y, Matsumoto M, Maruiwa H, Watanabe M, Nishizawa T: Intraspinal cyst communicating with the intervertebral disc in the lumbar spine: discal cyst. Spine 26:2112-2118, 2001

4. Chiu L, Tsai P, Chou P, Kuo T, Cheng C, Li M, et al.: A gas filled intradural cyst with intradural disc herniation: a case report. Acta Neurol Taiwan 17:36, 2008

5. Jeong G, Bendo J: Lumbar intervertebral disc cyst as a cause of radiculopathy. Spine J 3:242-246, 2003

6. Julius D, Basbaum AI: Molecular mechanisms of nociception. Nature 413:203-210, 2001

7. Kang B, Jung Y, Park S, Lee K: Discal cysts of the cervical spine in two dogs. J Vet Sci 4:543-545, 2015

8. Kawakami M, Tamaki T, Hayaslai N, Hashizunte H, Nislai H: Possible mechanism of painful radiculopathy in lumbar disc herniation. Clin Orthop Relat Res 351:241-251, 1998

9. Kim S, Ahn S, Choi G, Kim D: Discal cyst of the lumbar spine: a case report. Korean J Spine 2012; 9: 114-7.

10. Koga H, Yone K, Yamamoto T, Komiya S: Percutaneous CTguided puncture and steroid injection for the treatment of lumbar discal cyst: a case report. Spine 28:E212-216, 2003

11. Kono K, Nakamura H, Inoue Y, Okamura T, Shakudo M, Yamada $\mathrm{R}$ : Intraspinal extradural cysts communicating with adjacent herniated disks: imaging characteristics and possible pathogenesis. Am J Neuroradiol 20:1373-1377, 1999

12. Kwak H, Kim D, Kim T, Park H, Kim J, Jang J, et al.: Lumbar discal cyst causing bilateral radiculopathy. Surg Neurol Int 2:21, 2011

13. Marshman L, Benjamin J, David K, King A, Chawda S: "Disc cysts" and "posterior longitudinal ligament ganglion cysts": synonymous entities? Report of three cases and literature review. Neurosurgery $57: \mathrm{E} 818,2005$

14. Matsumoto M, Watanabe K, Tsuji T, Ishii K, Takaishi H, Naka- 
mura M, et al.: Microendoscopic resection of lumbar discal cysts. Minim Invasive Neurosurg 53:69-73, 2010

15. Saal J: The role of inflammation in lumbar pain. Spine 20:18211827, 1995

16. Takahashi H, Suguro T, Okazima Y, Motegi M, Okada Y, Kakiuchi T: Inflammatory cytokines in the herniated disc of the lumbar spine. Spine 21:218-224, 1996

17. Toyama Y, Kamata N, Matsumoto M, Nishizawa T, Koyanagi T, Suzuki N, et al.: Pathogenesis and diagnostic title of intraspinal cyst communicating with intervertebral disk in the lumbar spine. Rinsho Seikei Geka 32:393-400, 1997 